\title{
Melanoma stem cells - are there devils in the detail?
}

\section{Mark Shackleton ${ }^{1,2,3}$}

\author{
${ }^{1}$ Melanoma Research Laboratory and Department of Hematology \\ and Medical Oncology, Peter MacCallum Cancer Centre, East \\ Melbourne, Australia \\ ${ }^{2}$ Department of Pathology, Faculty of Medicine, Dentistry and \\ Health Sciences, University of Melbourne, Parkville, Australia \\ ${ }^{3}$ Howard Hughes Medical Institute, Life Sciences Institute, \\ Department of Internal Medicine and Center for Stem Cell Biology, \\ University of Michigan, Ann Arbor, Michigan, USA \\ Correspondance Mark Shackleton, e-mail: mark.shackleton@ \\ petermac.org
}

doi: $10.1111 / j .1755-148 X .2010 .00750 . x$

\section{Dear Sir,}

The importance of understanding melanoma progression is emphasized by the adverse clinical outcomes of most patients with advanced disease. Although the malignant nature of melanoma cells is determined by both genetic and epigenetic factors, considering the biological framework within which these factors develop is critical to identifying and targeting them for clinical benefit. If melanoma progresses according to a cancer stem cell (CSC) model in which rare tumorigenic cells not only renew their own malignant potential but also produce bulk populations of cells that are rendered irreversibly non-tumorigenic, then the implications for research and treatment of this disease would be profound. For example, it may be difficult to identify molecular drivers of tumorigenesis if tumorigenic cells are rare and not studied separately from less/non-tumorigenic melanoma cells that comprise most of the tumor. Moreover, if tumorigenic potential is present in only a small proportion of melanoma cells, then eliminating these cells will be essential to eradicate disease in patients. Because of this, much attention has been paid to the question of whether melanoma progresses according to a CSC model.

Over the last $5 \mathrm{yr}$, the pendulum of evidence has swung for and against the possibility that melanoma progresses according to a CSC model. Frank and colleagues tested the CSC model using classical in vivo techniques and found that only one in a million melanoma cells had tumorigenic potential that was $\sim 10$-fold enriched in cells expressing the membrane transporter molecule ABCB5 (Schatton et al., 2008). However, using more permissive in vivo tumorigenesis assay conditions, the Morrison laboratory subsequently identified tumorigenic potential in a very high proportion ( 25\%) of melanoma cells (Quintana et al., 2008). Since that study, Bosenberg and colleagues reported that tumorigenic cells can comprise a high proportion of cells in mouse melanomas (Held et al., 2010). These findings indicate that the frequency of tumorigenic melanoma cells had been previously underestimated by using tumorigenesis assays that unexpectedly concealed malignant potential in the vast majority of cells. It is critical in testing the cancer stem cell model to identify assay conditions that are permissive for revealing tumorigenic potential in cells (Shackleton and Quintana, 2010).

A recent study from the Weissman laboratory (Boiko et al., 2010) encourages melanoma biologists to re-evaluate the question of whether melanoma follows a CSC model. The central experiments of this paper that tested the CSC model were performed by isolating phenotypically distinct melanoma cells from tumors and transplanting them into immunocompromised mice. For tumors from which tumorigenesis by unfractionated melanoma cells was studied (\#213, \#114 and \#1119), limiting dilution analysis (Hu and Smyth, 2009) of the data presented in Supp Table 3 reveals that for all tumors the frequency of tumorigenic cells was $<0.05 \%$. This contrasts with the studies of malignant potential in unfractionated human melanoma cells by Quintana et al. (2008), in which the average frequency of tumorigenic cells was 25\% (Quintana et al., 2008; Fig 3c). As both studies evaluated tumors that were at similar stages of disease progression and that were obtained directly from patients, these data suggest that Boiko et al. (2010) used a tumorigenesis assay that was several orders of magnitude less sensitive than the assay used by Quintana et al. (2008).

Boiko et al. (2010) also found that cells expressing CD271 (also known as nerve growth factor receptor or p75) were significantly $(P<0.05)$ enriched in tumorigenic potential in $5 / 6$ of the melanomas studied directly from patients, compared with $\mathrm{CD} 271^{-}$cells. The finding of CD271 as a putative human melanoma stem cell marker contrasts with the findings of Held et al. (2010), who found that tumorigenic potential was enriched in the $\mathrm{CD} 271^{-}$fraction of mouse melanomas (Held et al., 2010). In addition, studies in the Morrison laboratory of melanoma cells obtained directly from multiple patients and separated according to CD271 expression have consistently grown tumors from transplants of low numbers of $\mathrm{CD}_{271^{-}}$cells, such that no enrichment of tumorigenic activity in $\mathrm{CD} 271^{+}$melanoma cells has been 
observed (E. Quintana, M. Shackleton, S. Morrison, unpublished).

The reasons for the contrasting results of Boiko et al. (2010) and Quintana et al. (2008) are not clear. Both studies evaluated melanomas at similar stages of disease progression [compare Suppl. Table 2, 'Sample tissue type, location' column, in Boiko et al. (2010) versus Suppl. Table 1 in Quintana et al. (2008)], so putative stage-related differences in tumor biology are not likely to explain the effect. Additionally, although Quintana et al. (2008) performed some experiments using cells obtained from tumors that had first been grown as xenografts in mice, the data were confirmed in non-xenografted melanomas obtained directly from six patients, including two primary cutaneous melanomas (Quintana et al., 2008; Fig 3c). The differences between the studies cannot therefore be explained by the use in Quintana et al. (2008) of cells only derived from xenografted melanomas.

A more likely explanation is the different tumorigenesis assay conditions used by each group. Boiko et al. (2010) used a one to three hour enzymatic digestion protocol to isolate cells from tumors before mixing sorted cells with standard Matrigel and transplanting intradermally into $\mathrm{Rag}^{-1-} \gamma \mathrm{c}^{-/-}(\mathrm{RG})$ mice. Quintana et al. (2008) used a much shorter (<30 min) digestion protocol with a different composition of enzymes and mixed sorted cells with high protein Matrigel before transplanting subcutaneously into NOD/SCID IL2R $\gamma^{-/-}$ (NSG) mice. Differences in tissue digestion methods may result in differences in the viability of cells and thus affect tumorigenic potential. Additionally, as the dermis is composed of much denser connective tissue elements than subcutaneous tissue, it is conceivable that intradermal injection may inhibit tumor formation by melanoma cells that are tumorigenic after subcutaneous injection. Also, it is possible that the host environment of $R G$ mice inhibits melanoma cell tumorigenicity in unexpected ways, despite these mice lacking B-, T- and NK cell function. Other differences between the tumorigenesis assays used may also contribute to the contrasting data. Although it would be surprising if any single assay variation explained the orders of magnitude difference in efficiency of tumor formation seen in the two studies, there can be large compounding effects of multiple assay differences on the detection of tumorigenic potential in human melanoma cells (Quintana et al., 2008).

Dramatically different interpretations of tumor biology can arise from seemingly minor differences in the detail of how tumorigenesis assays are performed (Quintana et al., 2008). If CD271- melanoma cells are non-tumorigenic only under certain assay conditions, it will be unwise to conclude that they do not contribute to disease progression in patients. It will be critical for other laboratories to test whether melanomas contain common or rare tumorigenic cells and to determine whether tumorigenic potential is restricted to phenotypically distinct cells that are hierarchically organized within tumors and distinguished by expression of CD271, ABCB5 or other markers. We encourage others to use sensitive assays to address these questions thoroughly, so that the full spectrum of cells with the potential to propagate disease can be detected. It will also be important to test carefully whether the contrasting findings of Quintana et al. (2008) and Boiko et al. (2010) can be explained by differences in the tumorigenesis assays used.

\section{References}

Boiko, A.D., Razorenova, O.V., van de Rijn, M. et al. (2010). Human melanoma-initiating cells express neural crest nerve growth factor receptor CD271. Nature 466, 133-137.

Held, M.A., Curley, D.P., Dankort, D., Mcmahon, M., Muthusamy, V., and Bosenberg, M.W. (2010). Characterization of melanoma cells capable of propagating tumors from a single cell. Cancer Res. 70, 388-397.

Hu, Y., and Smyth, G.K. (2009). ELDA: extreme limiting dilution analysis for comparing depleted and enriched populations in stem cell and other assays. J. Immunol. Methods 347, 70-78.

Quintana, E., Shackleton, M., Sabel, M.S., Fullen, D.R., Johnson, T.M., and Morrison, S.J. (2008). Efficient tumour formation by single human melanoma cells. Nature 456, 593-598.

Schatton, T., Murphy, G.F., Frank, N.Y. et al. (2008). Identification of cells initiating human melanomas. Nature 451, 345-349.

Shackleton, M., and Quintana, E. (2010). Progress in understanding melanoma propagation. Mol. Oncol. in press. doi 10.1016/ j.molonc.2010.06.006. 